\title{
Detection of ECG Fiducial Points Using Recursive Estimation and Kalman Filtering
}

\author{
Luis E Avendano ${ }^{1}$, Jorge I Padilla ${ }^{2}$, Edilson Delgado-Trejos ${ }^{3}$, David Cuesta-Frau ${ }^{4}$ \\ ${ }^{1}$ School of Electrical Technology, Universidad Tecnologica de Pereira, Colombia \\ ${ }^{2}$ Automatics Research Group, Universidad Autonoma de Manizales, Colombia \\ ${ }^{3}$ CM\&P Research Group, Instituto Tecnologico Metropolitano ITM, Colombia \\ ${ }^{4}$ Universitat Politecnica de Valencia, Alcoy Campus, 03801 Alcoy, Spain
}

\begin{abstract}
QRS complex detection is regarded as a baseline procedure for the segmentation of electrocardiographic (ECG) signals, as it is usually the most distinctive component of the signal. Unfortunately, many QRS detection algorithms do not work well in pathological heartbeats, where QRS morphology changes radically. This paper addresses QRS detection by using a novel approach based on recursive estimation of the QRS envelope using Kalman Filter and smoothness priors. This approach effectively estimates fiducial points, as it considers an interval-dependent adaptive threshold, which is independent of the heartbeat morphology, reaching a robust detection. In order to validate this proposal, the MIT-BH, QT, and ST-T databases were used. A global accuracy of $99.4 \%$ with a sensitivity of $96.9 \%$ was achieved. The experimental results demonstrated an improvement of the proposed Kalman filter, showing that the performance is stable, maintaining a high performance as the noise level increases.
\end{abstract}

\section{Introduction}

Commonly, from the $Q R S$ detection, a backward/forward search is carried out to find other components, i.e., $P$ wave, $T$-wave, and sometimes $U$-wave, in electrocardiographic (ECG) signal studies [1]. Likewise, the $Q R S$ detector is useful for obtaining the $R R$ interval, which analyzes heart rate variability (HRV), as the synchronization with the phonocardiographic signal, for several studies of arrhythmia [2-4] or heart murmur detection [5]. Accordingly, it is imperative that the $Q R S$ is detected from heterogeneous morphology; but, when the $Q R S$ complex changes radically, many detectors yield wrong results [6].

Onset, offset and peak location of ECG waves are known as fiducial points (FPs) [7]. Several algorithms for automatically detecting QRS complexes have been proposed; for instance, using empirical mode decomposi- tion [8], artificial neural networks [9], wavelets [10-13], reverse biorthogonal wavelet decomposition and nonlinear filtering [14], quadratic filtering [15], locally adaptive weighted total variation denoising [16], regular grammar and deterministic automata [17], combination of interval and trigonometric threshold values [6], and approaches for ultra-long-term ECG recordings [18]. The advantages of Kalman filter have been discussed in several studies regarding QRS complex detection [7,19]; however, the main problem is the initialization of both search locations and operating parameters.

This paper is focused on cases where the QRS complex morphology drastically changes due to pathologies related to severe arrhythmias. A Kalman filter approach uses recursive estimation routines associated with adaptive thresholding techniques, with the aim of improving the detection robustness independently of the heartbeat morphology. The algorithm parameter optimization is carried out on standard databases for comparing with other studies, using as evaluation criteria the values of accuracy and sensitivity.

\section{Proposed approach}

\subsection{Hybrid algorithm for QRS detection}

ECG signal can be described as a signal with additive noise, given by:

$$
y[k]=x[k]+s[k]
$$

where $y[k]$ is a noisy ECG signal, $x[k]$ is the known structure and $s[k]$ the unknown structure. Some types of disturbances (e.g., powerline interference and baseline wander) have a known basic structure, while a clean ECG signal can be modeled by the residual derived from the noisy signal and the estimated interference with time-varying variance. Thus, the interference can be estimated as the sum of $M$ basis functions, $\phi_{i}[k]$, multiplied by a set of time-varying 
coefficients $\alpha_{i}[k]$, expressed by:

$$
x[k]=\sum_{i=1}^{M} \alpha_{i}[k] \phi_{i}[k]
$$

This model (2) can be expressed by a state-space representation, as follows:

$$
\begin{aligned}
\boldsymbol{z}[k+1] & =\boldsymbol{A}[k] z[k]+\boldsymbol{B} v[k] \\
y[k] & =\boldsymbol{C}[k] z[k]+s[k]
\end{aligned}
$$

where, $z[k]$ is the state vector containing the dynamics expressed by $\alpha_{i}[k]$ and $\phi_{i}[k], \boldsymbol{A}[k]$ is the state transition matrix, and $\boldsymbol{C}[k]$ is the state measurement matrix. Thus, from expressions (1) and (3b), $x[k]=\boldsymbol{C}[k] z[k]$, where the interference dynamics is governed by (3a) and mixed with the clean ECG signal. The interference reduction can be interpreted as an estimation problem of unknown structures, which can be solved using Kalman filtering, as discussed in [20]. Then, a variance estimator of $\hat{s}[k]$ can be applied in order to determine the QRS complex envelope. This variance can be estimated using a smoothness priors method proposed in [21], where the noise model is obtained as a realization of white noise for $k=1, \ldots, N$ of $s[k] \approx \mathrm{N}\left(0, \sigma^{2}[k]\right)$ with unknown time-varying variance $\sigma^{2}[k]$. Using a transformation for $s[k]$, given by

$$
\chi^{2}[m]=\frac{1}{2}\left(s^{2}[2 m-1]+s^{2}[2 m]\right)
$$

A stochastic process $\chi^{2}[m]$ is achieved, which is an independent sequence of random variables with chi-square distribution and two freedom degrees of $\chi^{2}[m] \sim \chi_{2}^{2}$. Considering the following transformation

$$
t[m]=\ln \chi^{2}[m]+\gamma,
$$

where $\gamma=0.5772157$ is the Euler-Mascheroni constant, an independent random variable, $t[m]$, is generated with an almost normal distribution and moments, given by

$$
\mathrm{E}\{t[m]\}=\ln \sigma^{2}[m], \quad \sigma^{2}\{t[m]\}=\frac{\pi^{2}}{36}
$$

In order to obtain a smooth estimation of $\sigma^{2}[m]$, the $n$-th order difference equation should be considered for restricting the variance evolution, as follows

$$
\nabla^{k} t[m]=w[m]
$$

where $w[m] \sim \mathrm{N}\left(0, \tau^{2}\right)$ iid. Thus, the restriction model is

$$
\begin{aligned}
\boldsymbol{x}[m] & =\boldsymbol{F} \boldsymbol{x}[m-1]-\boldsymbol{G} w[m] \\
t[m] & =\boldsymbol{H} \boldsymbol{x}[m]+\boldsymbol{\xi}[m]
\end{aligned}
$$

Having $t[m]$, the envelope $b[k]$ can be recovered by

$$
b[k]=\exp (t[m / 2])
$$

This recursive estimation requires the signal resampling of $t[m]$, twice its sample rate.

\subsection{Adaptive threshold}

The final step of this approach consists of an intervaldependent threshold that can be updated at each detection for the $\theta_{i}$-time and remains fixed for the next interval until the threshold is exceeded and a new $Q R S$ is detected. This interval-dependent threshold structure $\eta_{I}[k]$ is based on the peak amplitude, updated exponentially from the $Q R S$ complex previously detected, as shown below:

$$
\begin{aligned}
\eta_{I}[k] & =\mu_{\tilde{e}_{e, i},} \quad k=\theta_{i}, \theta_{i+1}, \ldots \\
\tilde{z}_{e, i} & =\tilde{z}_{e, i-1}+\alpha\left(z\left(\theta_{i}\right)-\tilde{z}_{e, i-1}\right), \quad i \geq 1
\end{aligned}
$$

where $\tilde{z}_{e, i}$ is the exponential mean, and $z\left(\theta_{i}\right)$ represents the preprocessed signal amplitude of the more recently detected $Q R S$ complex in the time $\theta_{i}$. The $\mu$ parameter determines the fraction of the amplitude $\tilde{z}_{e, i}$ to be used in the threshold estimation and $\alpha$-parameter determines the rate with which the threshold may change.

\section{Experimental setup}

The hybrid algorithm for $Q R S$ detection consists of three stages (see Figure 1): conventional digital filtering, nonlinear transformation using the envelope detection algorithm based on Kalman filter and a decision rule.

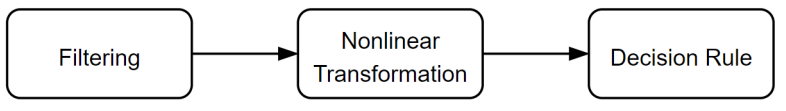

Figure 1. Experimental procedure stages

Algorithms were tested on three databases: MIT-BIH [22], QT [23], and European ST-T [24]. These properly labeled and validated databases provide reproducible and comparable results, with a large number of the most common ECG morphologies, as well as signals that are rarely observed, but clinically important. The proposed approach was compared with three approaches: a method based on moving-averaging incorporating with wavelet denoising [25], a detector based on the MaMeMi filter [26], and a detector based on dual-slope [27]. Firstly, Kalman filter parameters were adjusted in order to achieve a proper performance on the analyzed databases. In particular, the parameters $\tau^{2}$ for the envelope estimator and $\sigma^{2}$ under the powerline and baseline interference filter configurations. For both parameters, a scan between $10^{-3}$ and $10^{6}$ was performed, where the best performance was achieved for $\tau^{2}=1$ and $\sigma^{2}=10^{3}$. Next, ECG signals without interference were considered, and subsequently, the performance with powerline interference and baseline wander, using a range of signal to noise ratio (SNR) from $-12 d B$ to $12 d B$, was analyzed. Likewise, the effect of the parameter variation was studied, in order to generalize the properties of this approach in other scenarios. 


\section{Results}

Table 1 shows the performance of the hybrid-KF algorithm in terms of sensitivity for all the databases, comparing with the other three approaches. Results for powerline interference (S.PL.) and baseline wander (S.BL.) were considered. The best detection of fiducial points was achieved with the proposed approach. Figures 2 and 3 present accuracy results, taking both types of noise with different SNR levels. The hybrid-KF method (proposed approach) has a better performance in most of the SNR levels.

Table 1. Sensitivity results for all databases in $-6 d B$

\begin{tabular}{l|lll}
\hline \hline Database & Algorithm & S. PL. & S. BL. \\
\hline \hline \multirow{4}{*}{ MIT-BIH } & MA-Wavelet [25] & $99.5 \pm 0.5$ & $96.4 \pm 0.5$ \\
\cline { 2 - 4 } & MaMeMi [26] & $99.4 \pm 0.5$ & $96.7 \pm 0.3$ \\
\cline { 2 - 4 } & Dual Slope [27] & $99.7 \pm 0.3$ & $96.7 \pm 0.4$ \\
\cline { 2 - 4 } & This approach & $\mathbf{9 9 . 8} \pm \mathbf{0 . 2}$ & $\mathbf{9 6 . 8} \pm \mathbf{0 . 2}$ \\
\hline \hline \multirow{4}{*}{ QT } & MA-Wavelet [25] & $99.1 \pm 0.4$ & $96.2 \pm 0.4$ \\
\cline { 2 - 4 } & MaMeMi [26] & $99.3 \pm 0.4$ & $96.3 \pm 0.5$ \\
\cline { 2 - 4 } & Dual Slope [27] & $99.5 \pm 0.2$ & $96.1 \pm 0.5$ \\
\cline { 2 - 4 } & This approach & $\mathbf{9 9 . 6} \pm \mathbf{0 . 2}$ & $\mathbf{9 7 . 1} \pm \mathbf{0 . 2}$ \\
\hline \hline \multirow{4}{*}{ ST-T } & MA-Wavelet [25] & $99.3 \pm 0.2$ & $96.7 \pm 0.2$ \\
\cline { 2 - 4 } & MaMeMi [26] & $99.3 \pm 0.3$ & $96.5 \pm 0.3$ \\
\cline { 2 - 4 } & Dual Slope [27] & $99.5 \pm 0.3$ & $96.6 \pm 0.3$ \\
\cline { 2 - 4 } & This approach & $\mathbf{9 8 . 8} \pm \mathbf{0 . 2}$ & $\mathbf{9 6 . 7} \pm \mathbf{0 . 2}$ \\
\hline \hline
\end{tabular}

\section{Conclusion}

A $Q R S$ detection approach has been proposed based on recursive estimation of the envelope using the Kalman filter strengths. This approach effectively allows the estimation of fiducial points with similar or better performance than other well-known methods in the literature. It also allows real-time robust estimation. The $Q R S$ detector of this approach with interval-dependent threshold can be improved by using a time-dependent threshold, with the aim of rejecting large amplitude $T$-waves, allowing the detection of even low-amplitude ectopic beats. In general, the performance is consistent and stable in the presence of powerline and baseline wander noises, maintaining a high performance as the noise level increases.

\section{Acknowledgments}

The authors would like to thank the Doctorate Program "Tecnologias para la Salud y el Bienestar" - Universitat Politecnica de Valencia, Spain and AMYSOD Lab, Instituto Tecnologico Metropolitano ITM, Colombia.
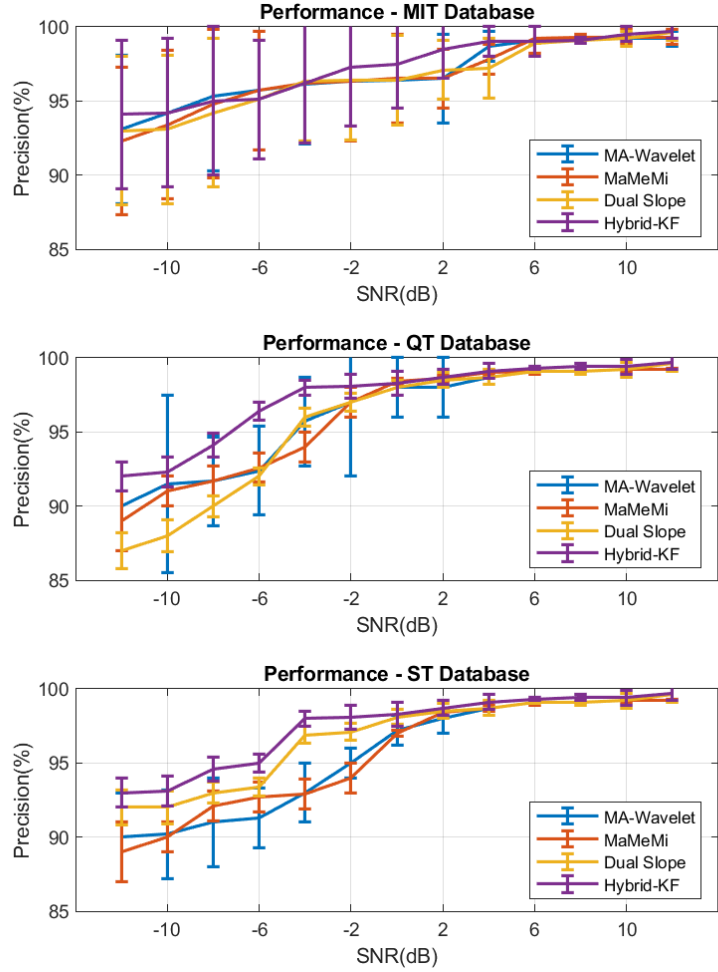

Figure 2. Accuracy results for baseline wander
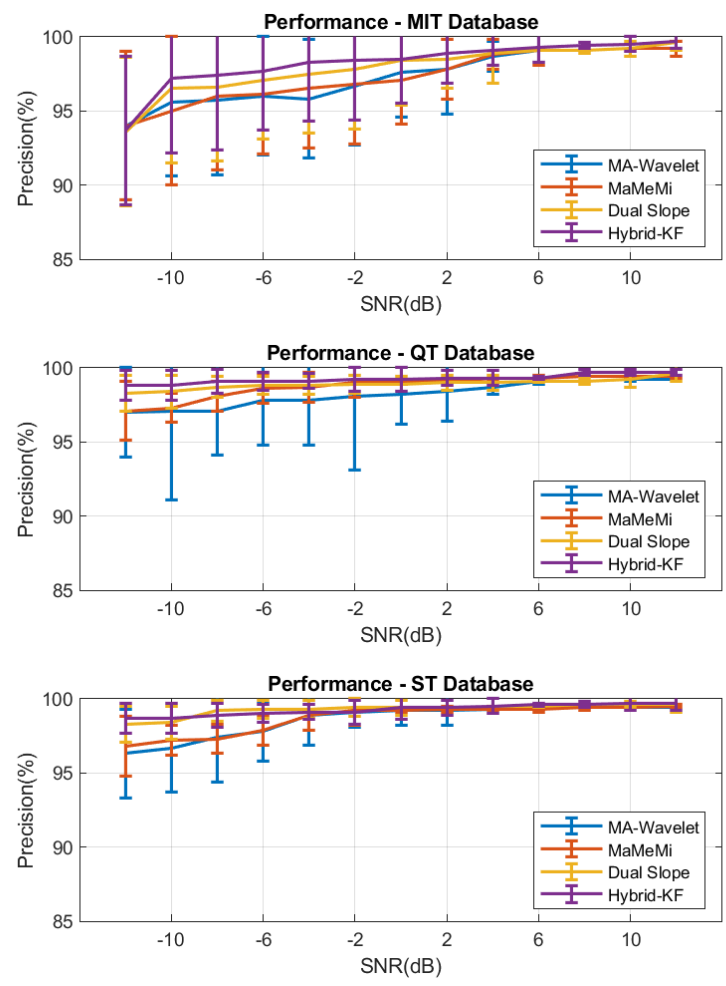

Figure 3. Accuracy results for powerline interference 


\section{References}

[1] Rodríguez-Sotelo JL, Delgado-Trejos E, Peluffo-Ordoñez D, Cuesta-Frau D, Castellanos-Domínguez G. WeightedPCA for unsupervised classification of cardiac arrhythmias. In 32nd Annual International Conference of the IEEE EMBS. 2010; 1906-1909.

[2] Delgado-Trejos E, Rodríguez JL, Jiménez F, Cuesta-Frau D, Castellanos G. Recognition of cardiac arrhythmias by means of beat clustering on ecg-holter records. In Computers in Cardiology, volume 34. 2007; 161-164.

[3] Becerra MA, Orrego DA, Mejía C, Delgado-Trejos E. Stochastic analysis and classification of 4-area cardiac auscultation signals using empirical mode decomposition and acoustic features. In Computing in Cardiology, volume 39. 2012; 529-532.

[4] Jiménez JA, Becerra MA, Delgado-Trejos E. Heart murmur detection using ensemble empirical mode decomposition and derivations of the mel-frequency cepstral coefficients on 4-area phonocardiographic signals. In Computing in Cardiology, volume 41. 2014; 493-496.

[5] Delgado-Trejos E, Quiceno-Manrique AF, Godino-Llorente JI, Blanco-Velasco M, Castellanos-Domínguez G. Digital auscultation analysis for heart murmur detection. Annals of Biomedical Engineering 2009;37(2):337-353.

[6] Mukhopadhyay SK, Krishnan S. Robust identification of QRS-complexes in electrocardiogram signals using a combination of interval and trigonometric threshold values. Biomedical Signal Processing and Control 2020; 61:102007.

[7] Akhbari M, Ghahjaverestana NM, Shamsollahi MB, Jutten C. ECG fiducial point extraction using switching Kalman filter. Computer Methods and Programs in Biomedicine 2018;157:129-136.

[8] Pal S, Mitra M. Empirical mode decomposition based ECG enhancement and QRS detection. Computers in Biology and Medicine 2012;42:83-92.

[9] Arbateni K, Bennia A. Sigmoidal radial basis function ANN for QRS complex detection. Neurocomputing 2014; 145:438-450.

[10] Chouakri SA, Bereksi-Reguig F, Taleb-Ahmed A. QRS complex detection based on multi wavelet packet decomposition. Applied Mathematics and Computation 2011; 217:9508-9525.

[11] Zidelmal Z, Amiroua A, Adnane M, Belouchrani A. QRS detection based on wavelet coefficients. Computer Methods and Programs in Biomedicine 2012;107:490-496.

[12] Yochum M, Renaud C, Jacquir S. Automatic detection of P, QRS and T patterns in 12 leads ECG signal based on CWT. Biomedical Signal Processing and Control 2016;25:46-52.

[13] Sharma A, Patidar S, Upadhyay A, Acharya UR. Accurate tunable-Q wavelet transform based method for QRS complex detection. Computers and Electrical Engineering 2019; 75:101-111.

[14] Mourad K, Fethi BR. Efficient automatic detection of QRS complexes in ECG signal based on reverse biorthogonal wavelet decomposition and nonlinear filtering. Measurement 2016;94:663-670.
[15] Phukpattaranont P. QRS detection algorithm based on the quadratic filter. Expert Systems with Applications 2015; 42:4867-4877.

[16] Sharma T, Sharma KK. QRS complex detection in ECG signals using locally adaptive weighted total variation denoising. Computers in Biology and Medicine 2017;87:187199.

[17] Hamdi S, Abdallah AB, Bedoui MH. A robust QRS complex detection using regular grammar and deterministic automata. Biomedical Signal Processing and Control 2018; 40:263-274.

[18] Malik J, Soliman EZ, Wu HT. An adaptive QRS detection algorithm for ultra-long-term ECG recordings. Journal of Electrocardiology 2020;60:165-171.

[19] Zhang Z, Yu Q, Zhang Q, Ning N, Li J. A Kalman filtering based adaptive threshold algorithm for QRS complex detection. Biomedical Signal Processing and Control 2020; 58:101827.

[20] Haykin S (ed.). Kalman Filtering and Neural Networks. Adaptive and Learning Systems for Signal Processing, Communications, and Control. John Wiley and Sons, 2001. ISBN 0-471-36998-5.

[21] Kitagawa G, Gersch W. A smoothness priors-time varying ar coefficient modeling of nonstationary covariance time series. IEEE Transactions on Automatic Control Jan 1985; 30(1): 48-56.

[22] MIT-BIH. Data base distribution, 1997. URL http: //ecg.mit.edu/.

[23] Laguna P, Mark RG, Goldberg A, Moody GB. A database for evaluation of algorithms for measurement of QT and other waveform intervals in the ECG. In Computers in Cardiology 1997. 1997; 673-676.

[24] Taddei A, Biagini A, Distante G, Mazzei MG, Pisani P, Rogerro N, Varanini M, Mark RG, Moody GB, Braaksma L, Zeelenberg C, Marchesi C. The european ST-T database: Development, distribution and use. Computers in Cardiology IEEE Computer Society Press Sep 1990; 177-180.

[25] Chen SW, Chen HC, Chan HL. A real-time QRS detection method based on moving-averaging incorporating with wavelet denoising. Computer methods and programs in biomedicine 07 2006;82:187-95.

[26] Castells-Rufas D, Carrabina J. Simple real-time QRS detector with the MaMeMi filter. Biomedical Signal Processing and Control August 2015;21: 137-145.

[27] Arefin MR, Fazel-Rezai R. Computationally efficient QRS detection analysis based on dual-slope method. In 2014 36th Annual International Conference of the IEEE Engineering in Medicine and Biology Society. 2014; 2274 2277.

Address for correspondence:

Jorge I. Padilla

Antigua Estacion del Ferrocarril, Universidad Autonoma de Manizales, Manizales-Colombia. Tel: (+57) 68727272 Ext. 146. E-mail: jorgei.padillabeautonoma.edu.co 